\title{
T-Cell Receptor Gamma-Delta
}

National Cancer Institute

\section{Source}

National Cancer Institute. T-Cell Receptor Gamma-Delta. NCI Thesaurus. Code C17432.

A heterodimeric complex comprised of the T-cell receptor gamma and delta chain proteins, which are linked by interchain disulfide bonds. This membrane bound complex is expressed on the surface of a small percentage of T-lymphocytes and is involved in the recognition of antigenic lipids, peptide antigens, and peptides presented by major histocompatibility (MHC) proteins. The gamma/delta dimer associates with the CD3 protein complex, which promotes ligand-dependent signaling. 